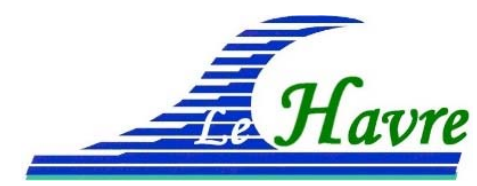

XVI ${ }^{\text {èmes }}$ Journées Nationales Génie Côtier - Génie Civil
Le Havre, 2020

DOI:10.5150/jngcgc.2020.050 (C) Editions Paralia CFL

disponible en ligne - http://www.paralia.fr - available online

\title{
Evaluation des protections en enrochements : inspection visuelle et diagnostic
}

\author{
Maxime DARBOT ${ }^{1}$, Vincent FUCHS ${ }^{1}$, Mikael DUMAS ${ }^{1}$
}

1. Compagnie Nationale du Rhône, Centre d'Analyse Comportementale des Ouvrages Hydrauliques, 4 rue de Chalon sur Saône, 69007 Lyon, France.

M.DARBOT@cnr.tm.fr ; V.FUCHS@cnr.tm.fr ; M.DUMAS@cnr.tm.fr

\section{Résumé :}

Les aménagements CNR du Rhône sont en service depuis 30 à 70 ans. Tout au long de cette période les revêtements de protections en enrochements ont subi des agressions d'origine hydraulique (courant, batillage et houle) et météorologique (gel, ensoleillement, ...) qui ont pu entraîner des dégradations. La maîtrise de la sûreté des ouvrages conjuguée à la maitrise des opérations de maintenance, a poussé CNR à engager une démarche d'études globales de diagnostic des protections en enrochements.

La méthodologie retenue consiste à déterminer la blocométrie des enrochements nécessaire et suffisante pour satisfaire aux conditions d'équilibres. Les conditions d'équilibres appliquées aux sollicitations hydrauliques du Rhône ont été fixées au travers d'une revue des pratiques internationales et des résultats d'essais en laboratoire sur modèles physiques. La blocométrie des enrochements est caractérisée en place à partir d'essais in situ (indice de continuité, essai de chute, blocométrie visuelle, ...).

Mots-clés :

Enrochements, Endiguements, Inspection visuelle, Diagnostic, Conception, Maintenance, Vieillissement, Batillage, Courant, Vent.

\section{Introduction et contexte}

CNR exploite près de $400 \mathrm{~km}$ d'endiguements répartis entre les retenues et les canaux d'amenée. Les enrochements assurent la protection des endiguements contre les sollicitations hydrauliques induites par la navigation, le vent ou encore le courant naturel du Rhône. La blocométrie généralement mise en place à l'origine correspondait à des enrochements 10/100 kg dans les zones normalement exposées et 100/400 kg dans les zones très exposées. La perte de la protection du parement amont constitue un des mécanismes précurseurs à une rupture de l'endiguement. Particulièrement dans le cas du Rhône où le courant peut atteindre 5 à $6 \mathrm{~m} / \mathrm{s}$ en crue et dont le trafic fluvial à grand gabarit génère du batillage tout au long de l'année.

A partir du début des années 1990, les protections des digues et berges du Rhône ont commencé à nécessiter d'importants travaux d'entretien, liés aux dégradations provoquées par le passage des bateaux, les crues, les vents violents et sur certains secteurs à une fracturation progressive des blocs d'enrochements émergés. Il était donc nécessaire 


\section{Thème 4 - Ouvrages portuaires, offshore et de plaisance}

d'examiner, avec des méthodes homogènes d'évaluations et de dimensionnement, l'état des revêtements. Ces revêtements en enrochements ont été conçus et réalisés sur une durée totale de 40 ans, avec des matériaux répondant à des normes internes de résistance. En effet, dans l'intérêt de garantir la qualité de ces enrochements, CNR a élaboré une procédure d'agrément de fourniture qu'elle impose aux carriers qui souhaitent fournir ses chantiers. De même, l'usage des matériaux de carrières proches du fleuve a été privilégié pour diminuer les coûts d'investissement liés notamment au transport.

A la fin des années 1990, CNR a engagé une série d'études diagnostics dans le but d'établir un plan pluriannuel d'entretien destiné à mettre en évidence les secteurs nécessitant réellement des renforcements.

Actuellement, il est nécessaire de mettre à jour ces diagnostics réalisés il y a plus de 20 ans en tenant compte de l'actualisation des données hydrauliques et des évolutions des méthodes de justification.

\section{Méthodologie du diagnostic}

La méthodologie consiste à confronter la blocométrie nécessaire pour satisfaire aux conditions d'équilibres des blocs à la blocométrie des enrochements en place.

\subsection{Caractérisation de la blocométrie nécessaire}

Les endiguements sont soumis à différentes sollicitations hydrauliques qui interviennent lors d'évènements exceptionnels (crues, vents) mais aussi en situation normale du fait de la navigation. Les principales sollicitations hydrauliques sont les suivantes :

- Le batillage, qui se caractérise lors du passage d'un bateau par un abaissement localisé du plan d'eau et la génération d'un système d'ondes se propageant en directions des berges ;

- Le vent qui forme des vagues à la surface de l'eau ;

- Le courant naturel qui peut atteindre des vitesses élevées lors des crues et qui est plus important dans les tracés courbes que dans les tracés rectilignes.

\subsubsection{Sollicitations induites par la navigation}

Le batillage correspond aux phénomènes induits par le passage des bateaux sur un plan d'eau. Un bateau qui navigue dans un canal est à l'origine de plusieurs sollicitations, provoquées par les ondes primaires (bourrelet de proue, courant de retour et onde transversale de poupe) et les ondes secondaires (ondes secondaires d'interférence).

Il existe également d'autres sollicitations de moindre importance telles que celles provoquées par les jets d'hélice. Elles font l'objet d'études spécifiques dans les secteurs où les bateaux accélèrent à partir de l'arrêt ou de la manœuvre à faible vitesse. Les composantes de l'agitation de l'eau au passage d'un bateau sont illustrées à travers la figure 1 . 


\section{XVİ̀mes Journées Nationales Génie Côtier - Génie Civil \\ Le Havre 2020}

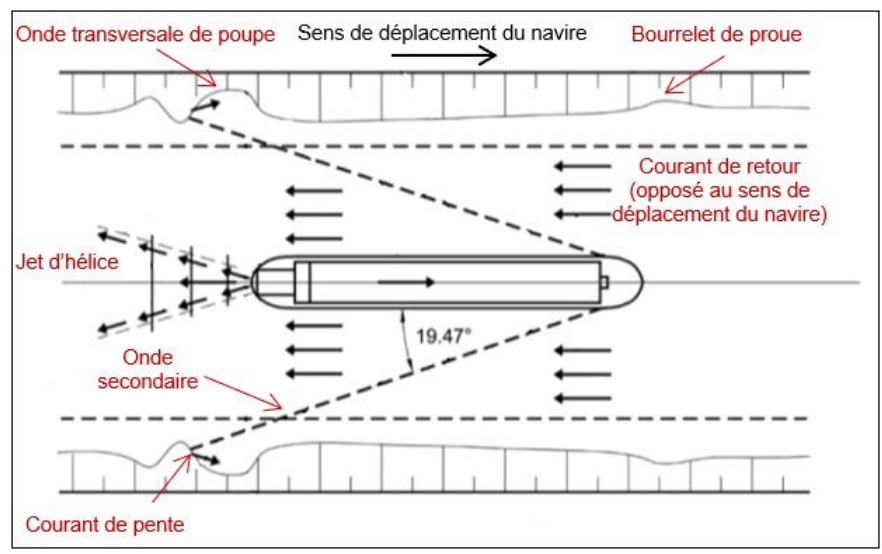

Figure 1. Agitation de l'eau induite par la navigation.

Au passage d'un bateau, le niveau d'eau commence par s'élever légèrement avant de décroitre au moment où le bateau passe au droit de l'observateur (bourrelet de proue). En avançant, le bateau pousse devant lui une masse d'eau qui, ne pouvant s'accumuler à l'avant du bateau, s'écoule de part et d'autre du bateau provoquant, par la mise en vitesse de cette eau, l'abaissement de la surface de l'eau (principe de Bernoulli) ainsi qu'un courant de retour associé.

Le niveau d'eau remonte (onde transversale de poupe) puis une série d'ondes atteint la berge et provoque les oscillations du plan d'eau (ondes secondaires). Un courant de pente se forme parallèlement aux rives lorsque les ondes de poupe se brisent ou lorsqu'elles sont de grande amplitude (PIANC, 2008).

L'action du batillage est proportionnelle à la vitesse de navigation des bateaux. Elle dépend de la géométrie de la voie d'eau, du type de bateau et de l'axe de navigation.

La méthode de justification retenue par CNR permet d'évaluer les différentes sollicitations hydrauliques dues au batillage. Elle offre un regard macroscopique et intégrateur des sollicitations. Ces hypothèses pourront être réévaluées sur les tronçons qui auront été identifiés comme problématiques pour tenir compte des spécificités locales (distance du chenal de navigation, conditions particulières de vitesse, etc.).

Un benchmark sur les pratiques internationales et des essais sur modèles physiques au Centre d'Analyse Comportementale des Ouvrages Hydrauliques (CACOH) de CNR ont permis de déterminer la méthodologie applicable aux retenues et canaux du Rhône :

- La vitesse de limite de navigation, l'abaissement du plan d'eau et le courant de retour associé sont ainsi calculés à partir de la théorie de Schijf (AIPCN, 1987);

- La hauteur des ondes de poupe et de proue sont estimées à partir de l'abaissement du plan d'eau en utilisant un simple coefficient (CIRIA et al., 2009). Le diamètre caractéristique des enrochements nécessaire pour résister à l'onde de poupe est calculé à partir de la formule de Laboyrie (AIPCN, 1987) ;

- Enfin, la formule de Havelock (SORENSEN, 1967) est utilisée pour calculer la hauteur des ondes secondaires d'interférence entre les ondes transversales de poupe et ondes 


\section{Thème 4 - Ouvrages portuaires, offshore et de plaisance}

divergentes de proue. Les coefficients permettant le calage de la formule de Havelock ont été validés sur modèles physiques au laboratoire de CNR (CNR, 1998). Le diamètre caractéristique des enrochements nécessaire afin de résister aux ondes secondaires d'interférences est calculé à partir de la formule de Verhey et Pilarczyk (AIPCN, 1987).

\subsubsection{Sollicitations induites par le vent}

L'action du vent a pour effet de soulever des vagues dont la hauteur dépend de la longueur du plan d'eau sur laquelle souffle le vent (Fetch), la hauteur d'eau et les caractéristiques du vent en lui-même (vitesse et durée).

Les situations de projets sont les mêmes que celles considérées pour les retenues de barrages, c'est-à-dire :

- Un vent de période de retour 1000 ans à la cote d'une retenue normale ;

- Un vent de période de retour 50 ans à la cote d'une retenue aux plus hautes eaux.

La vitesse moyenne du vent de période de retour donnée est déterminée suivant la norme EN 1991-1-4 (AFNOR, 2005).

La hauteur significative et la période des vagues sont calculées à partir des équations de Brettschneider (BAW, 2005). La génération de vagues dues au vent dans les retenues et les lacs est généralement limitée par la géométrie du plan d'eau. Pour les cours d'eau, le Fetch concerne parfois un linéaire assez faible qui n'est pas toujours représentatif de la longueur du plan d'eau réellement exposé au vent. Dans ce cas, on utilise la notion de Fetch effectif qui consiste à réaliser une moyenne des longueurs du plan d'eau autour du Fetch (voir figure 2). Le Fetch effectif est déterminé en traçant des lignes radiales à +/$45^{\circ}$ de la direction du vent considéré.

Un programme sous système d'information géographique (SIG) a été développé par CNR afin d'automatiser le calcul du Fetch effectif en tout point des endiguements du Rhône.

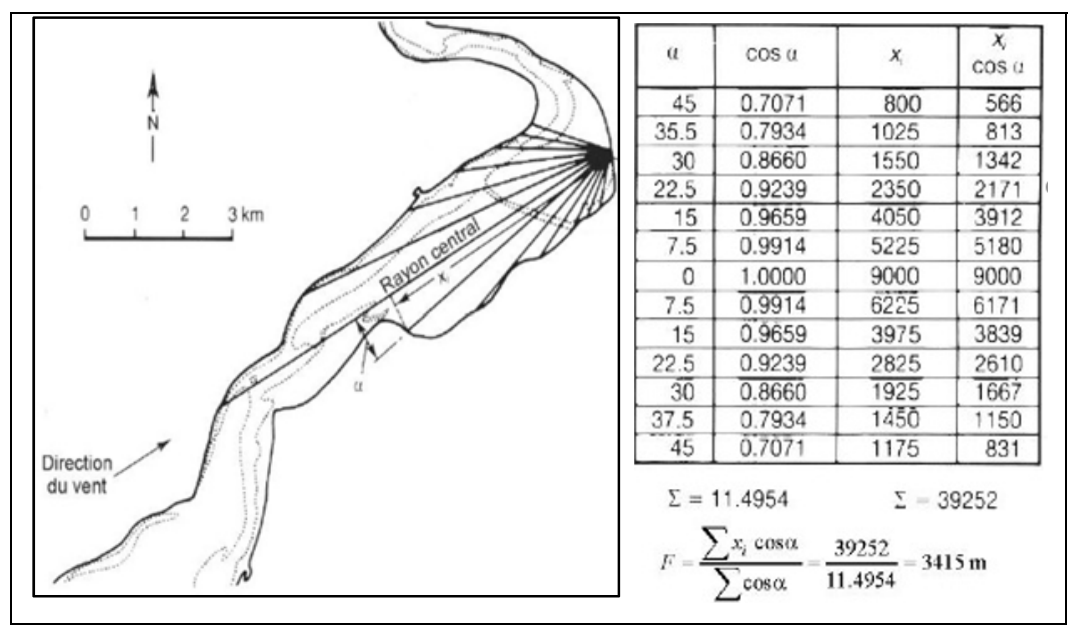

Figure 2. Représentation de la notion de Fetch effectif (CIRIA et al., 2009). 


\section{XVI'mes Journées Nationales Génie Côtier - Génie Civil \\ Le Havre 2020}

\subsubsection{Sollicitations induites par le courant naturel}

Un des objectifs d'une protection en enrochements est de protéger la berge contre l'action érosive du courant. Pour cela les blocs installés doivent rester en place même lorsque les vitesses d'écoulement du cours d'eau atteignent des valeurs exceptionnelles. Les formules de dimensionnement font intervenir principalement la vitesse des écoulements le long de la berge.

Les vitesses d'écoulement sont obtenues à partir d'études hydrauliques qui reposent classiquement sur les données issues d'un modèle mathématique. Chez CNR, le modèle utilisé est le modèle CRUE. Il s'agit d'un modèle $1 \mathrm{D}$ régulièrement remis à jour à partir notamment des relevés bathymétriques du Frédéric Mistral (bateau hydrographique de CNR) et des équipes territoriales.

En vue du dimensionnement des protections de berges, les résultats du modèle 1D aboutissant à des vitesses moyennées sur la section complète du fleuve apparaissent généralement suffisants pour obtenir des vitesses réalistes le long des berges en alignement droit. La modélisation 2D peut néanmoins s'avérer intéressante afin de disposer de vitesses plus raffinées contre les enrochements dans des cas particuliers, lors de travaux de réparation notamment.

Dans les secteurs en courbes, la vitesse des courants près des berges s'écarte de la vitesse moyenne sur la section. Dans ce cas, la vitesse moyenne est corrigée par un coefficient de survitesse. Ce coefficient dépend de l'exposition de la berge (intrados ou extrados de courbe) et du rapport entre le rayon de courbure de la berge et la largeur du plan d'eau. Pour le Rhône, les valeurs du coefficient de survitesse ont été validées sur modèle physique au sein du CACOH (CNR, 2000).

Une fois la vitesse d'écoulement connue, la stabilité de la protection en enrochements visà-vis de l'action des courants peut ainsi être déterminée à partir de la formule développée par le Laboratoire National d'Hydraulique sur la base de la formule de Ramette (RAMETTE, 1963). Cette dernière approche a également été validée par des essais sur modèle physique permettant de reproduire les écoulements rencontrés sur le Rhône. La formule de Ramette présente une très bonne concordance avec les résultats des essais. Afin d'être intégrateur dans le cadre de ses diagnostics à grande échelle, CNR applique un coefficient de 1,3 sur le diamètre caractéristique de l'enrochement.

\subsection{Caractérisation des blocs en place}

La caractérisation des blocs en place est fondée sur un plan d'échantillonnage par blocométrie sur chaque tronçon homogène. Ces tronçons homogènes sont définis en fonction de sollicitations hydrauliques proches déterminées pour la justification mais également sur le terrain en fonction de la blocométrie en place. L'essai blocométrique est réalisé suivant les principes des normes NF EN 13383 (AFNOR, 2003).

Cette inspection consiste à réaliser un état des lieux par des techniciens spécialisés en ingénierie des matériaux tous les $500 \mathrm{~m}$ et a minima 3 par tronçon homogène. Le contrôle 


\section{Thème 4 - Ouvrages portuaires, offshore et de plaisance}

est géoréférencé et permet d'avoir une vision de l'ensemble des protections en enrochements des endiguements de l'aménagement tout en donnant un avis qualitatif et quantitatif de la protection.

Cet état des lieux par blocométrie visuelle consiste à mesurer les trois dimensions de l'ensemble des blocs présents dans un carré de $3 \mathrm{~m}$ de côté. Ensuite, le poids médian des enrochements en place est calculé selon la procédure suivante :

- Mesure des trois dimensions du bloc : longueur, largeur, hauteur ;

- Calcul du volume puis de la masse du bloc ;

- Détermination du poids médian des enrochements à partir de la courbe blocométrique (voir figure 3).

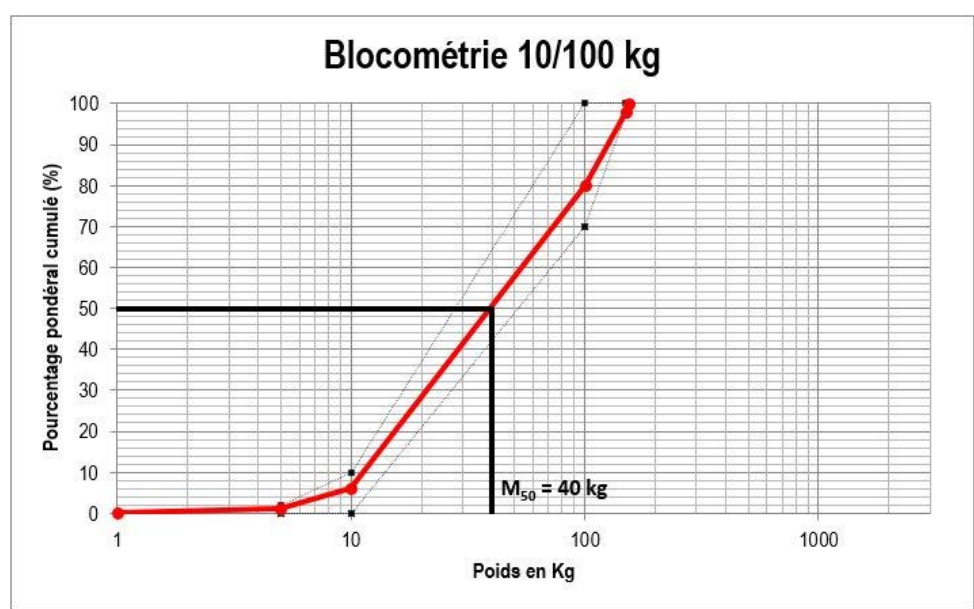

Figure 3. Représentation du pourcentage pondéral cumulé à $50 \%$ pour une blocométrie 10/100 kg.

Le poids médian des enrochements correspond au pourcentage pondéral cumulé égale à $50 \%\left(\mathrm{M}_{50}\right)$. L'essai blocométrique permet également de vérifier le respect d'un fuseau granulométrique au sein de la protection en enrochement.

\section{Résultats du diagnostic des aménagements de CNR}

Le diagnostic de l'état des revêtements en place se déroule en deux phases : une phase d'étude préalable permettant de caractériser les blocs nécessaires pour garantir les conditions de stabilité de la protection et une phase d'essais in situ permettant de caractériser les blocs en place.

Les résultats des essais réalisés sur site (essais blocométriques) sont comparés aux critères requis de stabilité des blocs (poids médian d'enrochements en $\mathrm{kg}$ nécessaire afin de résister aux différentes sollicitations hydrauliques). Cette comparaison met en évidence les secteurs conformes ou non par rapport aux calculs théoriques. Les résultats sont reportés sur SIG pour mettre en évidence les secteurs ne répondant pas à cette vérification générale. En cas de sous-dimensionnement apparent, un recalcul plus fin est réalisé en 


\section{XVI'̀mes Journées Nationales Génie Côtier - Génie Civil \\ Le Havre 2020}

intégrant notamment le coefficient multiplicateur réel de Ramette au tronçon voire des recalculs des sollicitations par modèles 2D, ainsi que des investigations terrain complémentaires notamment par des blocométries classiques par pesage complétées au besoin par des essais en laboratoire pour s'assurer des caractéristiques intrinsèques des roches (masse volumique notamment). Ces différentes phases d'analyses permettre de garantir la sûreté en identifiant les tronçons nécessitant une intervention.

Cette méthode a été appliqué sur plusieurs aménagements de CNR. Les études ont montré la grande difficulté à diagnostiquer les enrochements du fait d'un couvert végétal très développés sur les endiguements. Des sondages complémentaires devront être réalisés à l'aide d'une pelle mécanique. Les parements dont les enrochements ont pu être diagnostiqués présentent une blocométrie suffisante afin de résister aux sollicitations hydrauliques, mise à part sur certains secteurs où les enrochements étaient de moins bonnes qualités (migmatites, calcaires gélifs ou altérables qui provoque la diminution progressive de la taille des blocs d'enrochements au cours du temps). Les secteurs non conformes sont en cours de confortement afin de résister aux sollicitations hydrauliques.

\section{Conclusion}

CNR a mené un benchmark afin de déterminer la méthode la plus adaptée au diagnostic des protections en enrochements des endiguements du Rhône.

Pour chacune des sollicitations hydrauliques, les formules à retenir pour la justification de la stabilité des protections en enrochements ont été recherchées et adaptées si nécessaire au contexte spécifique du Rhône :

- Les formules permettant la justification contre les courants naturels ont été validées sur modèle physique et le choix de la formulation de Ramette corrigée par un facteur multiplicateur s'est avéré le plus pertinent;

- Concernant les effets du batillage, les formulations des protections à mettre en place sont complexes et très sensibles aux différents paramètres. L'analyse des différentes méthodes sur différents cas montre une variation importante des résultats. Pour donner suite à cette comparaison, la méthode retenue pour le Rhône est basée sur la théorie de Schijf pour les ondes primaires et la formule de Havelock pour les ondes secondaires ;

- En ce qui concerne les vagues générées par les vents, très peu de données sont disponibles sur ce sujet dans les canaux et rivières. Après vérification, CNR a décidé d'appliquer la notion de Fetch effectif habituellement utilisée pour les réservoirs. Cela permet de prendre en compte la largeur de fleuve sur laquelle le vent souffle.

La méthodologie finalement retenue a été appliquée à plusieurs aménagements de CNR. Les principaux résultats de ces applications montrent que les sollicitations hydrauliques sont modérées et les enrochements présents globalement aptes à résister aux sollicitations. Il est important de noter en retour d'expérience que la présence de végétation peut fortement limiter la visibilité des parements amont en enrochements et ainsi complexifier ces examens. Un entretien préalable peut donc s'avérer nécessaire. A noter également que 


\section{Thème 4 - Ouvrages portuaires, offshore et de plaisance}

cette méthodologie de diagnostic se veut intégratrice et rapide. Elle peut nécessiter des compléments d'analyse dans les cas où, via cette méthode, les résultats in situ ne seraient pas conformes aux calculs.

Enfin, en cas de nécessité de remplacement des enrochements en parement amont et étant donné ses besoins spécifiques en termes d'enjeux sûreté, CNR a élaboré en complément de la réglementation en vigueur, une procédure d'agrément de fourniture d'enrochements. Ce protocole d'agrément CNR a été présenté lors des JNGCGC de La Rochelle en 2018 (DUMAS, 2018).

\section{Références bibliographiques}

AFNOR (2003). Enrochements. NF EN 13383.

AFNOR (2005). Actions du vent. NF EN 1991-1-4.

AIPCN (1987). Recommandations pour le dimensionnement et la construction de revêtements souples incorporant des géotextiles pour les voies navigables intérieures. Rapport du groupe de travail $\mathrm{n}^{\circ} 4$ du Comité Technique Permanent. Bruxelles.

BAW -Bundesanstalt für Wasserbau- (2005). Principles for the design of bank and bottom protection for inland waterways. $200 \mathrm{p}$.

CIRIA, CUR, CETMEF (2009). Guide enrochement. L'utilisation des enrochements dans les ouvrages hydrauliques. Version française du Rock Manual. CETMEF, Compiègne, $38 \mathrm{p}$.

CNR -Compagnie Nationale du Rhône- (1998). Etude bibliographique. Dimensionnement des berges en enrochements pour résister au batillage. DI.LAB 98768, CNR, Lyon.

CNR -Compagnie Nationale du Rhône- (2000). Dimensionnement des protections des berges en enrochements dans les courbes du Rhône pour résister au courant. Etude sur modèles réduits. DI.LAB 00-206.

DUMAS M. (2018). Agrément technique de fournitures d'enrochement pour la mise en œuvre de carapaces de protection sur ouvrages hydrauliques. XV ${ }^{\text {èmes }}$ Journées Nationales Génie Côtier - Génie Civil, La Rochelle, pp 437-440. https://doi.org/10.5150/jngcgc.2018.049 PIANC (2008). Considerations to reduce environmental impacts of vessels. Report $n^{\circ} 99$ 2008. Bruxelles

RAMETTE M. (1963). Protection de talus au moyen d'enrochements. Bulletin du centre de recherches et d'essais de Chatou, $n^{\circ} 3$. EDF Chatou.

SORENSEN R.M. (1967). Investigation of ship-generated waves. Journal of the waterways and Harbors Division, Proceedings of the American Society of Civil Engineers, Vol 93(1), pp. 85-102. 